\title{
El marketing de servicios en la educación superior: "Nuestros alumnos, nuestros clientes".
}

Mg. Adm. Cynthia NUÑEZ ROSALES ${ }^{34}$

\section{RESUMEN:}

De acuerdo a Philip Kotler; El marketing de servicio considera que "Un servicio es cualquier actividad o beneficio que una parte puede ofrecer a otra. Es esencialmente intangible y no se puede poseer. Su producción no tiene porqué ligarse necesariamente a un producto físico". Todas las instituciones educativas, aerolíneas, bancos, entre otras empresas deben aplicar los principios de esta disciplina, porque tienen la responsabilidad de satisfacer a sus clientes. Rolando Arellano afirma que "El marketing es aquella especialidad que se ocupa de los procesos que buscan la satisfacción de las necesidades de los consumidores; no es necesario la transferencia de un bien hacia el cliente". Es por esto que el presente artículo brinda un enfoque sobre la trascendencia de considerar a nuestros alumnos; como clientes que difunden la calidad que brindan las instituciones educativas.

PALABRAS CLAVE: Marketing De Servicios, Educación, Satisfacción, Calidad, Alumnos.

\section{ABSTRACT:}

According to Philip Kotler; Marketing Service considers that "A service is any activity or benefit that one party can offer to another. It is essentially intangible and can not possess. Its production need not necessarily be linked to a physical product. "All educational institutions, airlines, banks, and other companies should apply the principles of this discipline, because they have the responsibility to satisfy their customers. Rolando Arellano said that "marketing is one specialty that deals with the processes that seek to satisfy the needs of consumers; transfer of goods to the customer is not required". That is why this article provides a focus on the importance of considering our students; as customers who spread the quality offered by educational institutions.

KEYWORDS: Marketing Services, Education, Satisfaction, Quality, Students.

34 Cualquier consulta sírvase por favor escribir a: cynthiamagister@gmail.com 
Las empresas del sector educativo superior como las universidades deben tener presente que también realizan "MARKETING DE SERVICIOS" cuando ponen énfasis en tomar a sus alumnos como los clientes y hacer todo lo posible que el nivel de satisfacción sobre los productos y los servicios que reciben sean altos.

En el marketing de servicios lo que comercializamos son productos intangibles, y para llegar al desarrollo de estos, primero investigamos a los consumidores reales y potenciales; para luego crear el producto y el servicio que satisfaga sus necesidades. En la educación existe también un mercado potencial, al cual las instituciones educativas superiores desean llegar a conquistar, tales como: egresados de colegios, alumnos de cuarto de secundarias, adultos trabajadores, etc. Aquí lo más importante, no es solo saber como atraerlos a nuestra institución educativa sino que cuando reciban el(los) producto(s) o servicio(s), este sea realmente tan buenos que nos recomienden y esto genere una mayor cantidad de nuevos clientes.

Es decir que como en toda empresa, hay detalles que recomiendo no descuidar, para lograr esta satisfacción:

- Los profesores deben subir su material educativo en sistemas virtuales o en todo caso llevarlo al aula para coordinar con los delegados respectivos y a su vez este con los alumnos, así mismo en el caso de copias para los cursos.

- Las instituciones educativas deben identificar las necesidades de capacitación, crear los programas de capacitación y exigir a los profesores que asistan semestral o anualmente. Muchos de estos programas deben estar vinculados a actualizaciones sobre la cátedra, metodología y estrategias de enseñanza y aprendizaje que pueden ser virtual, semi-presencial o presencial.

- Los docentes deben actualizar anualmente su hoja de legajo personal, presentando copias de los certificados de seminarios, cursos, congresos y/o diplomados relacionado a los temas que se dictan. (estas copias deben adjuntarse en su archivo personal).

De tal manera se persevera en la calidad de la docencia y si tiene cursos semi presenciales o virtuales:

- Las aulas virtuales poseen la capacidad de desarrollar espacios de aprendizaje usando la herramienta de chat, donde cada profesor debe participar por un tiempo mínimo de dos horas en ella y avisar a los alumnos previamente sobre las horas en las que estará conectado para que le realicen cualquier consulta o pregunta sobre los mismos temas o adicionales.

- Toda institución educativa debe desarrollar todo tipo de procedimientos didácticos escritos, visuales, virtuales, tecnológicos estrategias de enseñanza: instructivos, videos tutoriales, casos prácticos, etc.

- Siempre debe actualizarse la curricula de acuerdo a las necesidades de la demanda del mercado laboral. 
- En los cursos como Estadística; Proyectos de inversión; Ventas; Gestión Empresarial; Finanzas; Contabilidad, u otros dependiendo de la carrera debería incorporarse el uso de programas informáticos (software) tal como se utiliza actualmente en las diferentes empresas.

- Debemos brindar la oportunidad de capacitar a los docentes en cursos adicionales de informática aplicada a cursos de Estadística; Proyectos; Ventas; Gestión Empresarial; Finanzas; Contabilidad, etc. Por ejemplo, usar el SPSS en el Curso de Estadística; el Ms-Proyect en el curso de Proyectos; Excel y sus respectivas Macros para los cursos de Ventas; Finanzas; Gestión Empresarial; Contabilidad; etc.

"Se debe promover la coordinación entre los directores de escuela o coordinadores con los profesores de cátedra de manera permanente". Las relaciones entre los jefes y directores de la facultad con los docentes deben servir para verificar la ejecución de las siguientes actividades:

- Realizar una reunión de inicio de curso para indicarles que todo lo que se enseña debe ser aplicado a la realidad laboral.

- Hacer menciones o entrega de premios a los profesores por mejor desempeño durante un semestre.

- Entrega de un oficio para que puedan conocer el inicio del curso y de las diversas actividades planificadas durante todo el año y envió del syllabus propuesto.

- Permitir la participación de los docentes para la actualización de syllabus de acuerdo: a las normas del SUNEDU, al modelo pedagógico de la universidad, y de acuerdo al plan curricular de la carrera.

- Revisar y actualizar los exámenes a usarse en la evaluación discente.

- Revisar y actualizar las presentaciones a usarse para las clases magistrales y poder mejorar la calidad de la información o material a exponer, así como para permitir el mejor entendimiento de estos.

- Revisar, actualizar y propuesta de los libros para la consulta.

- Revisar las listas de los alumnos firmadas con las notas de los parciales y finales.

\section{Sobre la coordinación de la recuperación de clases}

El docente debe comunicar con anticipación a la coordinación y a los alumnos; la fecha y día de recuperación de clases, para que le puedan asignar un aula.

\section{Sobre la comunicación con los alumnos}

Se debe tener una mayor comunicación y consideración con el alumnado de parte de la dirección: 
- Cuando un profesor va a faltar informarles por mail o teléfono la falta de su docente con la debida anticipación para que el alumno no pierda su tiempo. Tengamos siempre presente que para todo alumno que trabaja y estudia el tiempo es muy valioso.

- Que la escuela busque un reemplazo para ese día. Pero el remplazo debe ser con un profesor que conozca a profundidad el tema a tratar.

\section{Materiales de apoyo para el curso.}

- Se debe brindar a los alumnos conexión con bibliotecas virtuales.

- Renovar libros de la biblioteca o crear un área de bibliotecas.

- Colgar Casos, Videos, Noticias Actualizadas, Videos complementarios acerca de la aplicación de los Temas a Estudiar. De esta manera los temas dejan de ser pura teoría.

\section{El ahorro de tiempo es importante y para esto debe utilizase las matrículas online.}

- Este servicio debe mejorar constantemente, porque hay personas que por su lejanía o la poca disponibilidad de tiempo, es más factible que se realice el Proceso de Matrícula de forma virtual.

\section{Apoyo a los estudiantes}

- La matrículas deben extenderse en el horario tarde (desde las 14:00 - 18:00 $\mathrm{pm})$ y noche teniendo presente que existe alumnos que trabajan (desde las 18:00-21:00 pm)

- Implementación de un sistema de tutoría, debido a que los alumnos desaprueban por diversas razones el curso, por lo que se debe tener una orientación para saber que hacer para nivelarse. Desde el profesor de la clase, al coordinador de la tutoría y por ultimo al profesor encargado de la tutoría debe desarrollar conversaciones planificadas que permitan indagar los motivos por los que desaprobó; ya que esta situación puede deberse al profesor, al método de enseñanza utilizado, a la falta de tiempo del alumno, etc. Se sugiere llevar un control o monitoreo continuo de los alumnos en mención. Por ejemplo el docente puede contar con un registro auxiliar digital para que se pueda actualizar automáticamente, realizar prácticas dirigidas para comprobar el aprendizaje obtenido y ejercicios continuos en clase. Debería existir la posibilidad de aumentar los créditos y poder matricularse en cursos que no tiene pre requisitos, pero dichos cursos deben ser de utilidad a la carrera que han escogido; ya que esto puede ser un motivo de desánimo para los alumnos.

- Las instituciones educativas deben preocuparse por el desarrollo extraordi- 
nario de los servicios que ofrecen, teniendo presente el alto nivel competitivo de las universidades nacionales e internacionales.

A continuación deseo aportar con algunas sugerencias que puestas en práctica pueden contribuir a desarrollar una ventaja competitiva interesante.

- Es recomendable contar con una plana docente estable, para ver las posibilidades de realizar publicidad a partir de su experiencia laboral, y poder comunicárselo a los alumnos, por ejemplo al matricularse.

- Lograr alcanzar una acreditación internacional.

- Realizar publicidad en diferentes medios de comunicación y redes sociales.

- Los profesores deben ser especializados y debe existir una práctica laboral sobre los cursos que dictan.

- Habilitar desde antes del primer día de clases, las aulas virtuales deberían para que los profesores, puedan colgar el material adecuado para su enseñanza.

- Permitir las prácticas pre profesionales para permitir el aprendizaje pragmático de la carrera.

Cuando una empresa se preocupa por cumplir lo que ofrece, el consumidor la considera honesta, todas estas sugerencias y recomendaciones son importantes para una institución educativa superior, para que pueda lograr un posicionamiento convincente en el competitivo mercado de la enseñanza superior.

\section{CONCLUSIONES}

1. La calidad en la atención al cliente representa una herramienta estratégica que permite ofrecer un valor añadido a los clientes con respecto a la oferta que realicen los competidores y lograr la percepción de diferencias en la oferta global de la empresa.

2. Las claves para lograr la calidad en el servicio son: tener clara la cultura empresarial, realizar una mejora continua y diseñar procesos para la solución de problemas que puede presentarse con los clientes.

3. El objetivo principal del proceso de producción del servicio es la satisfacción al cliente.

4. El rol principal del uso de la calidad, en la atención al cliente consiste en trabajar en las acciones de sensibilización y persuasión del resto de la empresa hacia el cliente.

5. Es importante tener presente la percepción que tiene el cliente, en este caso los alumnos sobre la institución educativa. 


\section{REFERENCIAS BIBLIOGRÁFICAS / WEBGRAFÍA}

1. DENTON, K. (1999). "Calidad en el Servicio a Los Clientes", Madrid, Editorial Díaz Santos S.A.

2. HOFFMAN, D. et al. (2002). "Fundamentos de Marketing de Servicios", Ediciones Paraninfo,

3. KOTLER, P. et al. (2004). "El Marketing de Servicios Profesionales". Barcelona. Paidos Ibérica.

4. LOVELOCK, Ch. (2004). "Mercadotecnia de Servicios". México. Prentice Hall.

5. ROSELLÓ, J. (2009). "Como Lograr una Excelente Atención Al Cliente". Madrid. Ediciones Internacionales Universitarias S.A.

6. www.marketingdeservicios.com/blog/categoria/mds/page/2/

7. http://sergiobernues.com/blog/el-nuevo-marketing-de-servicios/

8. http://politube.upv.es/play.php?vid=47021 